\title{
Solar cosmic rays by the ground level observation: anisotropy
}

\author{
Yury Balabin*, Aleksei Germanenko, Boris Gvozdevsky \\ Polar Geophysical Institute, 184209 Apatity, 26A Academgorodok, Russia.
}

\begin{abstract}
Nine large GLE events have been studied by the improved methodic of the inverse problem solution. The problems of the SCR anisotropy form setting have been in detail worked out in the new methodic. The SCR pitch-angle distribution form is automatically selected in solution search. It significantly improves the solutions convergence.
\end{abstract}

\section{Introduction}

Solar cosmic rays (SCR) reaching the earth's surface are rarely, and are called Ground Level Enhancements (GLE). Since 28.02.1942, when the first event (GLE01) took place, 72 events have been recorded. The measurements made at the ground level are still the basic ones, being, in fact, the only source of the data on the GLE events. The reason is that the GLE energy spectrum reaches at least the units of $\mathrm{GeV}$, and in case of large events, it reaches 20$30 \mathrm{GeV}$. Due to their high energy, SCRs are able to pass through the Earth's atmosphere and reach its surface, exerting, along the way, the ionization effect on the whole atmosphere. In so doing, the SCR intensity of large events increases that of the galactic cosmic rays flux by the order of 2-3. Correspondingly, penetrating the Earth's atmosphere, the SCRs exert great effect throughout its strata, from the ionosphere to troposphere and to the surface layer. In doing so, compared to galactic cosmic rays, SCRs have strong anisotropy, so, in calculating the SCR effect on the Earth and its atmosphere, it is necessary to determine not only the SCR spectrum but the exact SCR pitch-angle distribution (PAD).

\section{Instrumentation and methodic}

High energy cosmic rays are recorded by instruments placed only on the ground level. In addition, high SCR anisotropy needs a great number of detectors. The global neutron monitors (NM) network can be used to measure the SCR flux characteristics outside the Earth's magnetosphere. The SCR characteristics are the energy spectrum, anisotropy and PAD. These are derived by the inverse problem solution $[1,2]$. Asymptotic directions of acceptance were calculated with the Tsyganenko-03 magnetosphere model [3] and with 0.001 GV rigidity step by integrating the equation of a sample particle motion in the Earth's magnetosphere. The equations of motion are integrated by the Runge-Kutta method of the order of 4-5. Due to some peculiarities of the SCR recording methods, a neutron monitor has

${ }^{*}$ Corresponding author: balabin@pgia.ru 
a specific directivity pattern. In zenith angle increasing the solid angle of device reception increases together with the particles flow attenuation occurring due to the atmosphere absorption. The acceptance pattern reaches its maximum at $\sim 18^{\circ}$. The basic contribution is made on NM count rate by the particles passing at zenith angles of $10^{\circ}$ to $30^{\circ}$, i.e., at obliquecoming rather than vertical ones.

For the oblique-coming particles contribution to be taken into account correctly, as that of the NM, with the amount of these particles dominating, the NM angle pattern was divided into eight equal sectors, with the average directivity being specified in each sector. Thus, eight oblique cones defining the NM directivity pattern rather than one asymptomatic cone of vertical acceptance, were calculated for NM.

The new methodic also takes the peculiarities of high-mountain high-latitude stations into account. Due to the fact that the atmosphere layer over the NM in high-mountain areas is less, the directivity pattern maximum is shifted to the direction of wide angles, and the cutoff rigidity also decreases, widening the NM sensitivity range. Taking all the above said into account, we get a general equation to be used in calculating the response of the neutron monitor to SCR:

$$
I n c_{j}=\frac{1}{8} \cdot \sum_{(\varphi, \vartheta)=1}^{8} \sum_{R_{\min }}^{R_{\max }} J_{\|}(R) F(\theta(R)) S(R) A_{(\varphi, \vartheta)}(R) \Delta R
$$

where $\mathrm{Inc}_{\mathrm{j}}$ - is relative increase in the NM count rate at the station with the number of $\mathrm{j}$, $\mathrm{J}_{\|}(\mathrm{R})=\mathrm{J}_{0} \mathrm{R}-\gamma^{*}-$ is the differential spectrum of rigidity along the anisotropy axe, $\gamma^{*}=\gamma+\Delta \gamma \cdot(\mathrm{R}-1)$ where $\gamma$ - is the index of the power spectrum, $\Delta \gamma-$ is the increment index of $\gamma, \mathrm{J}_{0}\left(\mathrm{~m}^{-2} \times \mathrm{s}^{-1} \times \mathrm{srad}^{-1} \times \mathrm{GV}^{-1}\right)-$ is the normalization constant. The representation like this allows us to define the spectrum of a different form with only the help of 3 parameters: $\gamma, \Delta \gamma$, and $\mathrm{J}_{0}$. Other values in (1): $\mathrm{S}(\mathrm{R})$ - is the specific yield function connecting the flux of the primary particles of cosmic rays on the boundary of magnetosphere with the NM count rate [4]; $\theta(\mathrm{R})$ - is the pitch-angle at the specified rigidity $\mathrm{R}$ (the angle between the asymptotic direction of the specified rigidity $\mathrm{R}$ and the designed axis of anisotropy, which is specified by the coordinates $\Phi$ and $\Lambda$ in the system of GSE coordinates); the value $\mathrm{A}(\mathrm{R})=1$ is for the allowed trajectories and 0 is for the forbidden ones; $F(\theta(R))$ - is a modified pitch-angle distribution of SCR, which is in detail described below. The first sum in (1) takes into account the contribution made by all the 8 sectors, into which the NM acceptance pattern is divided, into the NM response. The second sum is the NM response through all the rigidities from $R_{\min }=1$ to $R_{\max }=20 \mathrm{GV}$, with the $\mathrm{R}=0.001 \mathrm{GV}$ rigidity step. The $\mathrm{NM}$ (1) responses are sequentially calculated at various values of the parameters of the anisotropic solar protons flux outside the Earth's magnetosphere boundaries $\left(\mathrm{J}_{0}, \gamma, \Delta \gamma, \mathrm{C}, \Phi, \Lambda\right)$ and a search is made of optimal; values of these parameters, under which the discrepancy is of minimum values.

\section{Features of pitch-angle distribution of SCR}

In the SCR distribution, two processes are dominating, such as particles flare focusing in the spreading out magnetic field and scattering on magnetic inhomogeneity. The pitch-angle scattering of the particles in the interplanetary magnetic field (IMF) is due to the inhomogeneities that induce the distortion of the first adiabatic invariant. The heterogeneities character may greatly differ in various events and is determined by the Sun's activity, providing wide variety of the PAD types. The simplest PAD is produced if the wavelength of the particle is constant in IMF and the angle of scattering is independent of the pitch-angle. In this case the PAD form is close to the Gaussian like [5].

What is more, SCRs can be defined within the model not always with the particles flux from one direction (from the Sun). A reversed flux is also possible (toward the Sun) if the Earth is in the loop structures of the interplanetary magnetic field, which takes place rather 
often [6-8]. In this case, being captured by the magnetic loop, SCRs reach the bottom of the loop, are reflected and move in the reverse direction. On the Earth in his case, a flux is observed in two directions: one moves from the Sun, the other (reflected) - to the Sun. The situation like this is called a two-directional one. In addition, the particles with a wide pitchangle have a small speed projection along the force line (with the pitch-angle of $90^{\circ}$, the particle moves around the circumference and perpendicular to the force line, without shifting along the force line). So, the particles with wide pitch-angles come to the Earth with a 30minute delay if compared to the particles with small pitch-angles, i.e., in PAD at the initial stage of the event there is observed a gap in wide pitch-angles.

Based on the visual analysis of the NM data, it is impossible to determine the presence of the reverse flux or the gap. So, it is necessary to use the form of PAD specifying and the parameters so that, in searching an optimal inverse problem solution, the specifying of the PAD form would be carried out automatically and a priori (just before the obtaining the results), and its various forms would be the function of the parameters values.

The importance of the true specifying the PAD form is in that, in inverse problem solving, all the parameters are equal. If the distribution form specified (one-directional flux, twodirectional flux, the flux with the gap in pitch-angles near $90^{\circ}$ ) does not correspond to that being in fact present in the GLR (for instance, a two-directional flux is impossible to be defined by simple Gaussian), then, in searching the inverse problem solution, firstly, there will be found no solution with small discrepancy, secondly, great discrepancy will be compensated at the expense of the change in the SCR spectrum parameters. Then, before the solution starts, it is necessary to have a notion of what form of PAD the SCRs have. Or it is necessary to specify the form (function) so that, changing the parameters, it would be possible to specify various forms. The main requirement to this function is that it should contain minimum number of additional parameters. The addition of new parameters into (1) results in less stability of the solution.

The least number of parameters in (1) in case of a one-directional flux is 5. As it was said above, these are $(\gamma, \Delta \gamma, \mathrm{C}, \Lambda$ and $\Phi)$. In case of a two-directional flux, three parameters more are added. A special function (2) has been produced, which, being increased by only two parameters, allows defining in fact any PAD forms: simple Gaussian (a one-directional flux), doubled Gaussian (a two-directional flux), gaussian with gap on pitch-angles close to $90^{\circ}$, and a linear PAD is also available [5], as well as other forms.

A new PAD function is an equation:

$$
F(\theta(R)) \sim \exp \left(-\frac{\theta^{2}(R)}{C}\right) \cdot\left(1-a \cdot \exp \left[-\frac{(\theta(R)-\pi / 2)^{2}}{b}\right]\right)
$$

where $\mathrm{C}-$ is the PAD width, $\mathrm{a}$ and $\mathrm{b}$ - are two constants allowing us to vary the form of distribution through very wide ranges. If parameter $\mathrm{a}>0$, then PAD will have a gap at the pitch-angles of about $90^{\circ}$, the value a $<0$ provides a hump at these pitch-angles. With $\mathrm{a}=0$, the PAD form turns into a simple Gaussian, which is in fact determined by the first factor. According to the further study of (2), under some combinations of the values $(C, a, b)$, it is possible to produce other forms: a linearly-decreasing form, a pedestal-form - a sum of isotropic and anisotropic fluxes, and others. The advantage of (2) is that, if it is introduced into (1), only two parameters are added and the number of PAD forms specified is great. As for the inverse problem considered earlier, it was solved with the fixed PAD form.

One more aspect should be noted here. During a GLE-event, the PAD form can cardinally change. It may be due to both the PAD in the source at the Sun and the conditions of distribution in the interplanetary magnetic field (IPMF). Secondly, the SCR flux contains the particles of different energy - from $1 \mathrm{GV}$ to tens of GV. In case of heterogeneities in IPMF, the SCR distribution will depend also on energy. In addition, a number of studies show that there are observed two components of SCRs in the GLE events such as prompt and delay components $[9,10]$ having different diffusion lengths. These reasons determine a complicated and changeable nature of PADs, which is observed near the Earth. In case when 
a GLE-event is studied in detail, the SCR parameters are determined during the event at definite points in time.

In this case, it is in fact impossible to specify a PAD form for each point in time manually, and the PAD form selected for the initial stage, may happen to be quite unsuitable at the phase of the maximum or of the decreasing. A new form (2) suits perfectly to determine PAD during the consistent points in time during of the event because it automatically fits to the actual situations on the base of the data recorded at the stations.

\section{A new PAD model consistence with the experimental data}

Figure 1 shows the comparison between two inverse problem solutions. In the first case, PAD in (1) was specified in a particular form (simple Gaussian, without the second factor in (2)); in the second case - in a complete form of (2), in which it is possible to obtain an arbitrary form by search of the parameters values $(\mathrm{C}, \mathrm{a}, \mathrm{b})$. The Gaussian function used in the first case, is not able to a gap in PAD at the pitch-angles close to $90^{\circ}$, and the solution obtained has a great discrepancy for NM, whose cones of acceptance are turned from the Sun: the calculation of these NMs response resulted in strong discrepancy with the factual data obtained at these NMs. In some GLE events, the SCRs are a collimated flux of relativistic protons [11]. When the flare reaches an area on the Earth, a strong SCR effect is observed there, outside the flare, the flux is absent and no effect is observed.
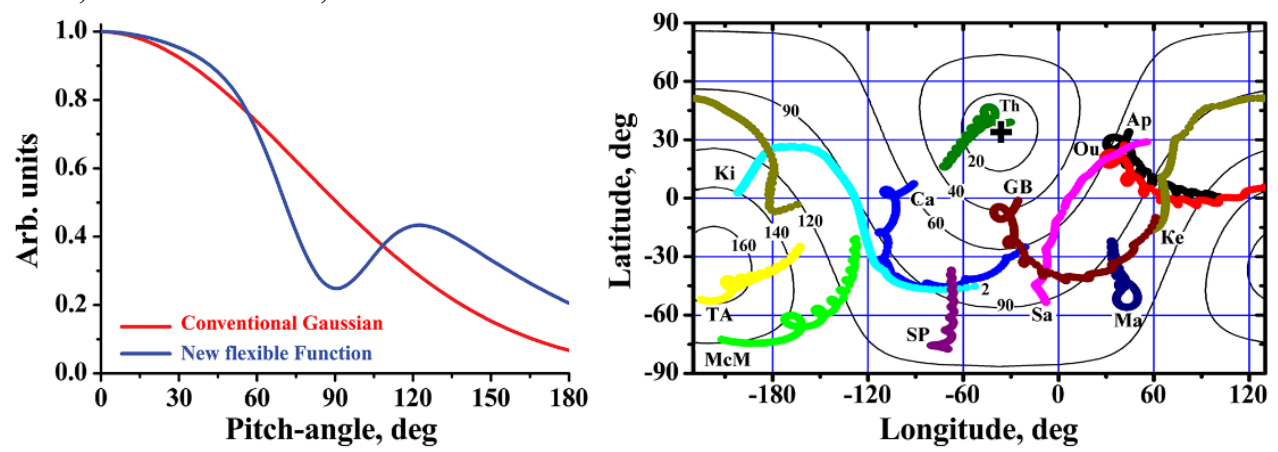

Fig.1. Left. The PAD forms specified after the inverse problem solution, for event GLE59, in specifying PAD in Gaussian, using a new function (2). Right. A map of asymptotic cones of aceptance at a number of stations for event GLE59. Abbreviations in the map: Ki - Kingston, TA - Terre Adelie, McM McMurdo, Ca - Calgary, SP - South Pole, Th - Thule, GB - Goose Bay, Sa - SANAE, Ou - Oulu, Ma - Mawson, Ap - Apatity, Ke - Kerguelen.

As shown in Fig. 1, the PAD failure at the pitch-angles close to $90^{\circ}$ will result in much less intensity at the stations whose cones of receiving are within the band of the pitch-angles of $70^{\circ}-110^{\circ}$. In Fig. 2, these are the stations of Kingston, Calgary, SANAE, Goose Bay, Mawson. As we see, to find the true solution of an inverse problem, it is necessary to use a flexible form of PAD that could be fitted to the actual situation in the process of the solution search. The PAD form not suitable for the actual one will result in a wrong calculation of the response at many stations and, in the long run, will significantly worsen the solution convergence.

Making a comparison between the intensity profiles at a pair of stations whose cones are compact and are in the key intervals, we make sure in that PAD has peculiarity at the pitchangles of about $90^{\circ}$. One of these is close to the axis of anisotropy, another is within the band mentioned, at $70^{\circ}-110^{\circ}$ from the axis. Fig. 2 shows an example of the same event - GLE59. The cone of Tule is in fact on the axis of anisotropy. The cone of the station of South Pole is within the band mentioned (the map of asymptotic cones in Fig.1, right). At the phase of 
growth, at 10:45 UT PAD had a significant gap at the pitch-angles close to $90^{\circ}$. The amplitudes of growth recorded at the stations differ at this period of time approximately threefold. The similar difference is between the values of PAD for the pitch-angles of $0^{\circ}-30^{\circ}$ and $70^{\circ}-110^{\circ}$ (curve 1). At the later phase of GLE59 in PAD, one can see a "hump" at the pitch-angles close to $90^{\circ}$, in the profiles, South Pole exceeds Thule. The excess is less than 1.5 , the same difference is in PAD. That is, the PAD forms obtained in the course of the inverse problem solution, show the factual differences between the intensity profiles at the NM stations. It means that in numerical modeling the SCR travelling through the Earth's atmosphere and in calculating the interaction between them, it is necessary to correctly take into account not only the SCR spectrum obtained in inverse GLE-problem solving but also PAD and the position of the axis of anisotropy.

The SCR flux parameters determined at the consistent points of time allow us to observe the dynamics of its spectrum and PAD, and to distinguish the characteristic phases.
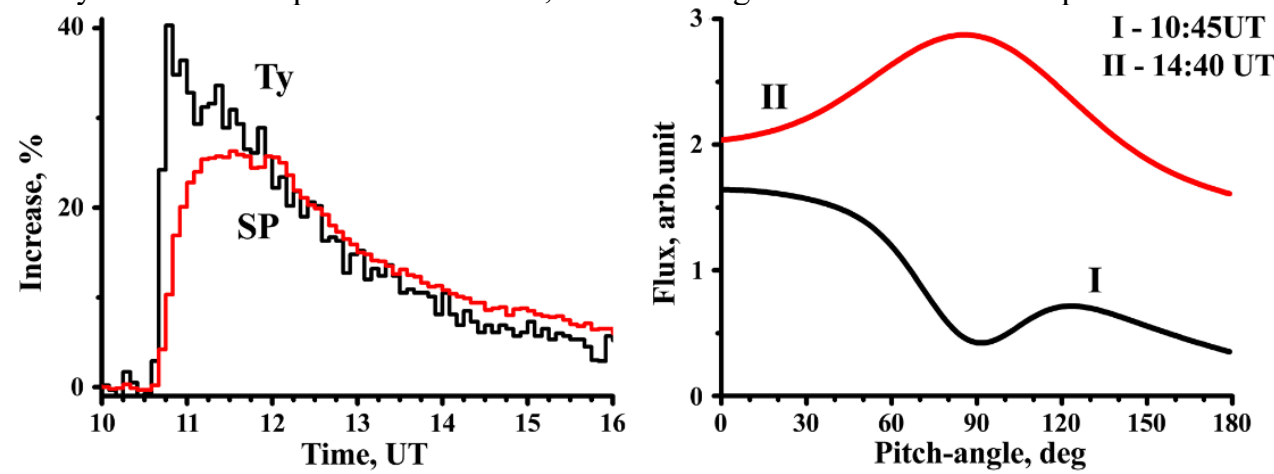

Fig.2. An example showing the presence of the peculiarity in PAD at the pitch-angles close to $90^{\circ}$ in event GLE59 during different points of time, and the reflection of these peculiarities in the intensity profiles. Left. The intensity profiles at two key stations: - Thule (Th) and South Pole (SP). Right. The PAD form for the phase of growth (I) and decreasing (II) .It is far from being typical and cannot be defined by simple Gaussian.
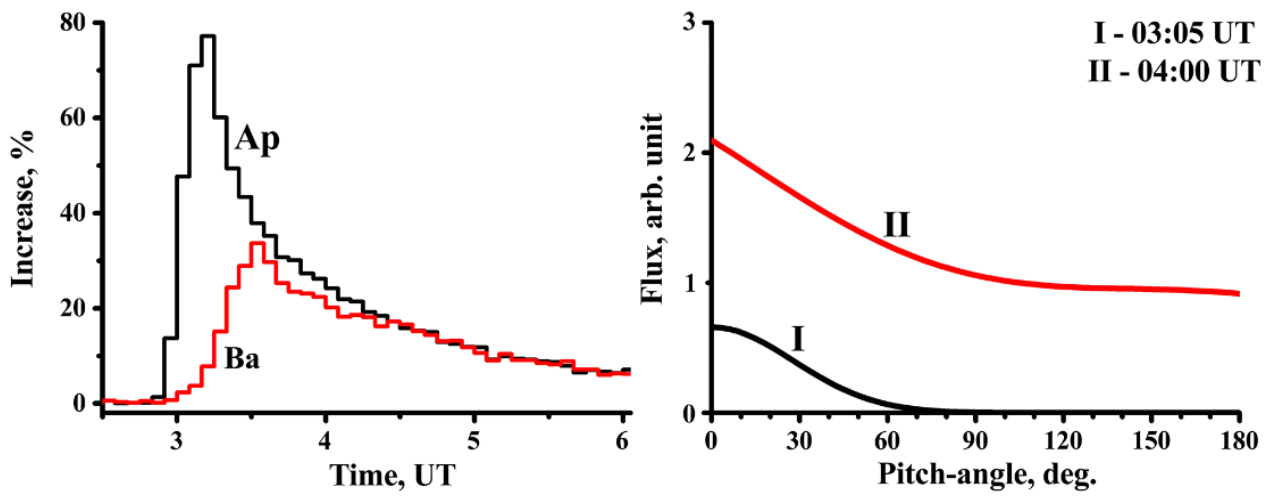

Fig.3. Left. A specific form of PAD in event GLE70. Left. The intensity profiles at the stations of Apatity and Barentsburg. Right. PAD for various points of time. At the initial phase, the SCR flux was rather narrow, within the interval of $30-40^{\circ}$. Later, at the phase of decreasing, it came to consist of two parts: an isotropic part (pedestal) and a linearly descending part at pitch-angles of less than $90^{\circ}$.

One more characteristic example is event GLE70 (Fig.3). The event is rather unnatural. It occurred on 13.12.2006, when the Sun's activity was close to its minimum, and the interplanetary medium was calm, the vector of IMF was almost in the plane of ecliptics.

All in all, nine GLE events were processed, with a new form of PAD specifying. The 
introduction of a new flexible form of PAD specifying improved the discrepancy in inverse problem solving one-and-a half-two times. In so doing, the SCR spectrum obtained in a new solution, differs slightly from the previous one, while PAD-forms differ significantly. New PAD-forms in events GLE show great variety, reflecting the peculiarities of the interplanetary medium state in each event.

\section{Conclusion}

This paper presents the result of modification of the methodic for the GLE inverse problem solution. According to the new methodic, the functional dependence of the pitchangle distribution of the SCR flux is specified implicitly. The functional PAD form is formed by introducing two new parameters, in consistency with the actual data. The necessity to use this method to specify the PAD form is due to intensive SCR anisotropy and to that the PAD form is inclined to change in the course of one event.

The study has been supported by the RSF grant 18-77-10018

\section{References}

1. Miroshnichenko L.I., Klein K.L., Trottet G., Lantos P., Vashenyuk E.V., Balabin Yu.V., Gvozdevsky B.B., J. Geophys. Res., 110, A09S08 (2005)

2. Vashenyuk E.V., Balabin Yu.V., Gvozdevsky B.B., Astrophys. Space Sci. Trans., 8, 1 (2011)

3. Tsyganenko N.A., J. Geophys. Res., 107, A8, 1176 (2002)

4. Debrunner H., Flueckiger E., 8th European Cosmic Ray Symp., Rome (1984)

5. Bieber J.W., Evenson P.A., J. Geophys. Res., 91, 8713 (1986)

6. Ivanov V.E., Obridko V.N., Shelting B.D., Astronomy reports., 41, 2 (1997)

7. Manoharan P.K., Kundu M.R. Astrophys. J., 592 (2003)

8. Shatten K.H., Mullan D.J. J. Geophys. Res., 72, 5609 (1977)

9. Vashenyuk E.V., Balabin Yu.V., Miroshnichenko L.I., Adv. Space Res., 41, 926 (2008)

10. Perez-Peraza J.A., Galiegos-Crus A., Vashenyuk E.V., Miroshnichenko L.I., Geomagnetism and Aeronomy, 32, 2 (1992)

11. Timashkov D.A., Balabin Yu.V., Barbashina N.S., $\quad$ Vashenyuk E.V., Kompaniyets K.G., Mannokki D.Zh., Petrukhin A.A., Saavedra O., Trinkero D.Zh., Shutenko V.V., Yashin I.I., Publ.H. RAN. Series Physical, 73, 3 (2009) 